\title{
Frequency Synchronization Analysis in Digital lock-in Methods for Bio-impedance Determination
}

\author{
Robert Brajkovič ${ }^{1}$, Tomaž Žagar ${ }^{2}$ and Dejan Križaj ${ }^{1}$ \\ ${ }^{1}$ University of Ljubljana, Faculty of Electrical Engineering, Tržaška 25, 1000 Ljubljana, Slovenia \\ ${ }^{2}$ Geodetic Institute of Slovenia, Jamova cesta 2, 1000 Ljubljana, Slovenia, rbrajkovic@gmail.com
}

\begin{abstract}
The lock-in method is one of the most frequently used methods for reconstruction of measured signals and as such frequently applied in the (bio)impedance method to determine the modulus and phase of the (bio)impedance. In implementation of the method in a (bio)impedance measurement device one has to consider possible non synchronized frequencies of the reference and the analyzed signals as well as potential sources of noise. In this work we analyzed these errors theoretically and experimentally. We show that both amplitude and phase errors depend on the relative difference of the frequencies of the reference and investigated signal as well as the number of integration periods. Theoretically, these errors vanish during the determination of the (bio)impedance modulus and phase. In practical implementation the inaccuracies appear at points of very low determined signal amplitudes due to the limited accuracy of analog to digital converters and are distributed around these points due to other sources of noise inherent in implementation of the measurement device.
\end{abstract}

Keywords: Impedance, bioimpedance measurements, frequency synchronization, lock-in method.

\section{INTRODUCTION}

$\mathrm{I}^{\mathrm{N}}$ N PHYSICAL EXPERIMENTS we often analyze periodic signals of small amplitudes that are buried in noise and other signal disturbances with amplitudes (energy) that might be significantly larger than the analyzed signal itself. Because conventional filtering techniques do not provide satisfactory results in such cases, special filtering techniques are required in order to differentiate signal from noise. One of the most frequently used methods uses the so called lock-in approach (also known as phase sensitive detection) [1], [7]. Phase sensitive detectors require (pre) knowledge of a period of analyzed periodic signal. A reference signal is created in accordance with an expected measured signal and is compared with it. Depending on the implementation of the lock-in method there are several possibilities of generation of a reference signal as well as its synchronization with the measured signal [11]. In this investigation we will analyze the problems of synchronization or rather non-synchronization of the external reference signal and the measured signal in particular in the frame of its usage in the (bio)impedance measurement method. In cases where a signal is accompanied by broad band noise it is feasible to generate reference signal externally instead of using excitation signal as reference. In this case the phase sensitive detector responds also to harmonic components of the excitation signal even though the fundamental component is well defined [11]. Bioimpedance as defined in the electrical circuit theory is essentially a transfer impedance, determined as a ratio of a voltage and a current complexor which are not necessarily related to the same nodes [5]. However, the technique assumes that the same current flows through all the nodes. This method is particularly of interest in studies of impedance methods in biological systems in order to reduce or even eliminate the difficulties related to the complex electrode-tissue electrical phenomena [10].
The discussed topics will be analyzed theoretically as well as experimentally using a portable battery powered bioimpedance measuring device that is currently under development [8]. Depending on the required application bioimpedance measuring devices can vary significantly. Here our goal was to develop a low cost portable device with a frequency range from $1 \mathrm{~Hz}$ to $40 \mathrm{kHz}$ to be used in BIA analysis. The device is based on usage of a low cost microcontroller with built-in AD/DA converters, which are used to generate the sinusoidal driving (input) signal and to perform acquisition of the system response. The data is transferred using a $\mathrm{WiFi}$ protocol to the laptop computer on which the digital lock-in method is performed and the bioimpedance is determined [8]. Several problems might arise from using low-cost, low-resolution AD/DA converters not optimized for real-time data acquisition. For example sinusoidal driving signal is limited by the 10 bit resolution where the data acquisition is prone to jitter and other sources of noise. Internal or intrinsic sources of noise are inherent in a circuit while external sources refer to the noise present in the signals applied or to the noise introduced into the circuit by some other means [2], [17]. Another problem is the prerequisite for signal generation based on exact whole number of points in a period [14]. Also, a clock frequency of a microcontroller may vary in time [6], which in our case results in slight adjustment of a frequency of the driving signal. Frequency drift describes the change in accuracy of a given frequency to environmental changes such as temperature, humidity, pressure or simply changes over long periods of time [18]. These circumstances together with sources of noise inherent in the electronic design and device realization require detailed analysis of the error related to not precisely tuned frequencies of the reference and the driving signal.

Here we will first briefly describe the fundamental principle of the lock-in method that is based on the properties of the correlation function. Then we will show 
how this is implemented in the (bio)impedance method. Next, non-synchronization is analyzed mathematically and experimentally. Finally, we demonstrate how the nonsynchronization influences determination of the (bio)impedance.

\section{THEORETICAL INVESTIGATION}

Correlation between two signals can be used as a measure for similarities between the two signals [15]. In case two signals have the same period $T_{0}$ the average crosscorrelation is determined as

$$
R_{12}\left(\varphi, T_{0}\right)=\frac{1}{T_{0}} \int_{-T_{0} / 2}^{T_{0} / 2} f_{1}(t) f_{2}(t+\varphi) d t
$$

Signals $f_{1}(t)$ and $f_{2}(t+\varphi)$ may differ but are related by having the same shape and the frequency (period) of the input signal. They are shifted in time, which is denoted through a phase difference $\varphi$. A schematic description of the lock-in method based on correlation techniques is presented in Fig.1. Clearly, if the signals are not correlated the cross-correlation function is zero and is non-zero otherwise. Signal response depends on the degree of correlation between the measured signal and reference [11].

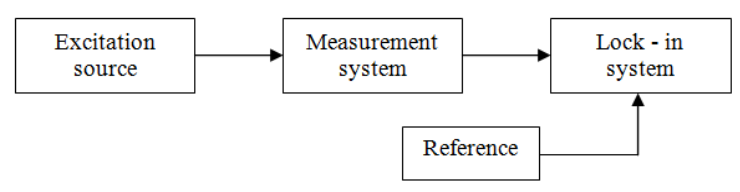

Fig.1. Basic principle of the lock-in method.

\section{1. (Single) phase sensitive detection}

Phase sensitive detection is based on two operations: synchronous demodulation and integration. First, the input signal is multiplied with the reference one and then it is integrated (low pass filtered) resulting in a DC signal $v(t)$ as shown in Fig.2.

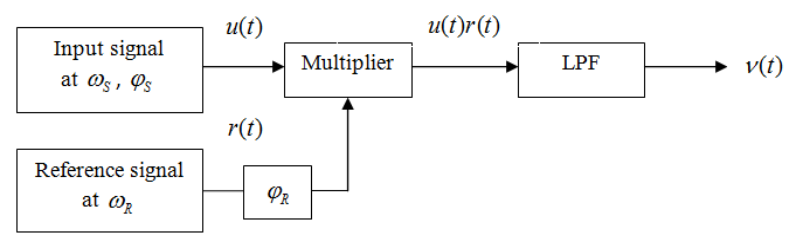

Fig.2. Schematic description of the phase sensitive detection.

Due to the properties of the correlation functions, the phase sensitive detection behaves as a band pass filter with respect to its reference frequency [12]. The bandwidth of the filter depends on the integration time (number of integrated cycles $N$ ). If the integration is carried out over $N$ periods of the sinusoidal perturbation, the equivalent filter selectivity is given by [4]

$$
\frac{\Delta \omega}{\omega_{R}}=\frac{1}{N}
$$

where $\omega_{R}$ is the center frequency and $\Delta \omega$ is the bandwidth.

In case the measured (and the reference) signals are sinusoidal - as they are in the (bio)impedance method - the input signal can be written as

$$
u(t)=U_{S} \sin \left(\omega_{S} t+\varphi_{S}\right)
$$

where $U_{S}, \omega_{S}$ and $\varphi_{S}$ are the amplitude, frequency and the phase of the signal, respectively. Likewise, the reference signal is written as

$$
r(t)=U_{R} \sin \left(\omega_{R} t+\varphi_{R}\right)
$$

Multiplication of the two signals yields

$$
\begin{aligned}
& u(t) r(t)=\frac{1}{2} U_{S} U_{R} \cos \left(\left[\omega_{S}-\omega_{R}\right] t+\varphi_{S}-\varphi_{R}\right)- \\
& -\frac{1}{2} U_{S} U_{R} \cos \left(\left[\omega_{S}+\omega_{R}\right] t+\varphi_{S}+\varphi_{R}\right)
\end{aligned}
$$

The first term with $\left[\omega_{S}-\omega_{R}\right]$ is attributed to the low frequencies and the second term with $\left[\omega_{S}+\omega_{R}\right]$ to high frequencies of the multiplied signal.

In case of equal frequencies of the reference and input signal $\left(\omega_{R}=\omega_{S}\right)$, the multiplication result is simplified to

$$
\begin{aligned}
& \left.v_{1}(t)\right|_{\omega_{R}=\omega_{S}}=u(t) r(t)= \\
& =\frac{1}{2} U_{S} U_{R} \cos (\phi)-\frac{1}{2} U_{S} U_{R} \cos \left(2 \omega_{S} t+\varphi_{S}+\varphi_{R}\right)
\end{aligned}
$$

where $\phi=\varphi_{S}-\varphi_{R}$. A well-known result is obtained when the result is averaged over all measured periods $\frac{1}{N T} \int_{0}^{N T} v(t) d t$ yielding only a DC component

$$
\left.\overline{v_{1}(t)}\right|_{\omega_{R}=\omega_{S}}=\frac{1}{2} U_{S} U_{R} \cos (\phi)
$$

The output expresses two properties: linearity and sensitivity. The output is maximal when the reference and the input signals are in phase. If the amplitudes of the signals are fixed, the output depends on the cosine of the phase difference $\phi=\varphi_{S}-\varphi_{R}$. This is named phase detection [1].

In case the term $\cos (\phi)$ is constant the output is linear and depends only on the amplitudes $U_{R}$ and $U_{S}$ which is known as amplitude detection.

In case the frequencies of the reference and input signals differ, the multiplication yields 


$$
\begin{aligned}
& \left.v_{1}(t)\right|_{\omega_{R}>\omega_{S}}=\frac{1}{2} U_{S} U_{R} \cos \left(\phi-\Delta \omega_{S} t\right)- \\
& -\frac{1}{2} U_{S} U_{R} \cos \left(2 \omega_{S} t+\Delta \omega_{S} t+\varphi_{S}+\varphi_{R}\right)
\end{aligned}
$$

with $\omega_{R}=\omega_{S}+\Delta \omega_{S}$. Integration of both parts does not result in a DC component proportional to $\cos (\phi)$ but in a much more complex term depending on the ratio $\frac{\Delta \omega_{S}}{\omega_{S}}$, the phase difference $\phi=\varphi_{S}-\varphi_{R}$ and the number of integration periods $N$ :

$$
\left.\overline{v_{1}(t)}\right|_{\omega_{R} \diamond \omega_{S}}=\frac{1}{2} U_{S} U_{R}\left[\begin{array}{l}
-\frac{1}{2 \pi N} \frac{\omega_{S}}{\Delta \omega_{S}}\left[\sin \left(\phi-2 \pi N \frac{\Delta \omega_{S}}{\omega_{S}}\right)-\sin \phi\right]+ \\
+\frac{1}{4 \pi N}\left[\sin \left(4 \pi N+2 \pi N \frac{\Delta \omega_{S}}{\omega_{S}}+\phi\right)-\sin \phi\right]
\end{array}\right]
$$

Non-synchronization between the reference and the input signal thus yields phase and amplitude determination errors. For instance, the phase determination errors (due to solely non-synchronized frequencies of the signals) can be calculated as $\eta\left[^{\circ}\right]=\left.\phi\right|_{\omega_{R}=\omega_{S}}-\arccos \left(\left.2 \overline{v_{1}(t)}\right|_{\omega_{R}<>\omega_{S}}\right)$ and are presented in Fig.3 for several different phase differences. Phase error is dependent on term $\arccos \left(\left.2 \overline{v_{1}(t)}\right|_{\omega_{R}<>\omega_{S}}\right)$, which approaches limit value of $90^{\circ}$ with increasing ratio $\frac{\Delta \omega_{S}}{\omega_{S}}$.

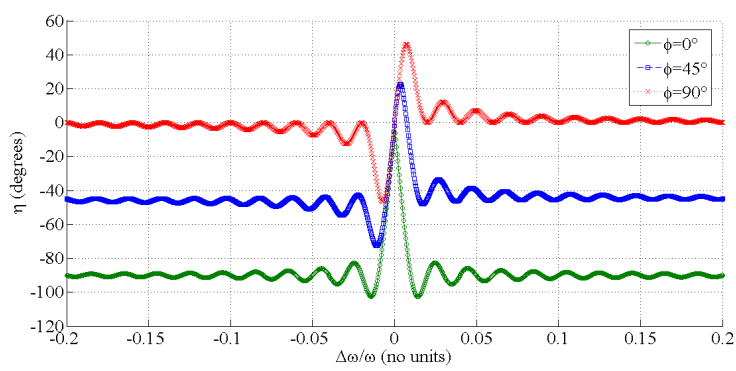

Fig.3. Phase difference determination depending on $\frac{\Delta \omega_{\mathrm{S}}}{\omega_{\mathrm{S}}}$ for

$$
N=50 \text { and phase differences } \phi=0^{\circ}, 45^{\circ}, 90^{\circ} \text {. }
$$

\subsection{Two phase sensitive detection}

The two phase technique of the lock-in method in the (bio)impedance method is most often implemented as shown in Fig.4. This method eliminates the difficulties of phase dependency in phase sensitive detection. The response is therefore maximal when the input and reference signals are brought in-phase at the phase sensitive detector input. This incorporates adjusting the setting of the phase shifter very carefully to $\phi=\varphi_{S}-\varphi_{R}=0^{\circ}$, which is in particular sensitive in case of the noisy input signal with a low amplitude. This phase sensitivity is reduced by the usage of a pair of phase sensitive detectors operated in quadrature. This arrangement enables both the in-phase and quadrature components of a coherent signal to be measured simultaneously [11].

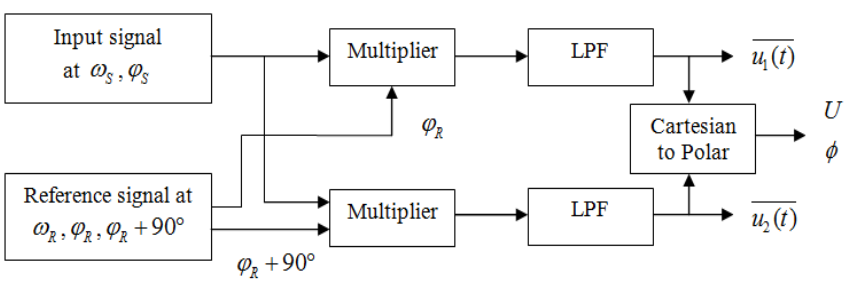

Fig.4. Schematic presentation of the two phase lock-in system.

For synchronized input and reference signals $\left(\omega_{R}=\omega_{S}\right)$ the outputs are

$$
\left.\overline{u_{1}(t)}\right|_{\omega_{R}=\omega_{S}}=\frac{1}{2} U_{S} U_{R} \cos (\phi)
$$

and

$$
\left.\overline{u_{2}(t)}\right|_{\omega_{R}=\omega_{S}}=\frac{1}{2} U_{S} U_{R} \sin (\phi)
$$

where $\left.\overline{u_{1}(t)}\right|_{\omega_{R}=\omega_{S}}$ denotes the 'in phase' or real and $\left.\overline{u_{2}(t)}\right|_{\omega_{R}=\omega_{S}}$ the 'quadrature' or imaginary component. The magnitude of the input signal can be determined from

$$
\left.U\right|_{\omega_{R}=\omega_{S}}=\sqrt{\left(\left.\overline{u_{1}}\right|_{\omega_{R}=\omega_{S}} ^{2}+\left.\overline{u_{2}}\right|_{\omega_{R}=\omega_{S}} ^{2}\right)}
$$

eliminating the phase dependence. The phase is then determined as

$$
\left.\phi\right|_{\omega_{R}=\omega_{S}}=\tan ^{-1}\left(\left.\overline{\overline{u_{2}}}\right|_{\omega_{R}=\omega_{S}}\right)
$$

For non-synchronized frequencies we get

$$
\left.\overline{u_{1}(t)}\right|_{\omega_{R} \diamond \omega_{S}}=\frac{1}{2} U_{S} U_{R}\left[\begin{array}{l}
-\frac{1}{2 \pi N} \frac{\omega_{S}}{\Delta \omega_{S}}\left[\sin \left(\phi-2 \pi N \frac{\Delta \omega_{S}}{\omega_{S}}\right)-\sin \phi\right]+ \\
+\frac{1}{4 \pi N}\left[\sin \left(4 \pi N+2 \pi N \frac{\Delta \omega_{S}}{\omega_{S}}+\phi\right)-\sin \phi\right]
\end{array}\right]
$$

and

$$
\left.\overline{u_{2}(t)}\right|_{\omega_{R} \diamond \omega_{S}}=\frac{1}{2} U_{S} U_{R}\left[\begin{array}{l}
\frac{1}{2 \pi N} \frac{\omega_{S}}{\Delta \omega_{S}}\left[\cos \left(\phi-2 \pi N \frac{\Delta \omega_{S}}{\omega_{S}}\right)-\cos \phi\right]- \\
-\frac{1}{4 \pi N}\left[\cos \left(4 \pi N+2 \pi N \frac{\Delta \omega_{S}}{\omega_{S}}+\phi\right)-\cos \phi\right]
\end{array}\right]
$$

In case the amplitude and the phase of the input signal is determined using the same procedure as for synchronized signals ((12) and (13)), this results in phase and amplitude determination errors. 
The relative amplitude error is determined as

$$
\xi[\%]=\frac{\left.U\right|_{\omega_{R}=\omega_{S}}-\left.U\right|_{\omega_{R}>\omega_{S}}}{\left.U\right|_{\omega_{R}=\omega_{S}}} \cdot 100 \% .
$$

It is presented in Fig. 5 for $N=50$ (chosen purely for figure clarity reasons). Clearly, the error is zero at $\omega_{R}=\omega_{S}$ and non-zero in other cases.

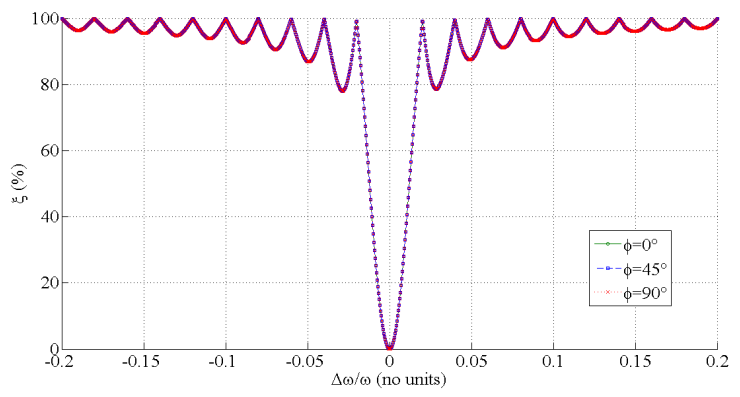

Fig.5. Relative amplitude determination error depending on $\frac{\Delta \omega_{\mathrm{S}}}{\omega_{\mathrm{S}}}$

$$
\text { for } N=50 \text {. }
$$

The phase determination error is determined as

$$
\lambda\left[^{\circ}\right]=\left.\phi\right|_{\omega_{R}=\omega_{S}}-\left.\phi\right|_{\omega_{R} \triangleright \omega_{S}}=\tan ^{-1}\left(\frac{\left.\overline{u_{2}}\right|_{\omega_{R}=\omega_{S}}}{\left.\overline{u_{1}}\right|_{\omega_{R}=\omega_{S}}}\right)-\tan ^{-1}\left(\frac{\left.\overline{u_{2}}\right|_{\omega_{R} \odot \omega_{S}}}{\overline{u_{1} \mid \omega_{R} \curvearrowright \omega_{S}}}\right)
$$

and is shown in Fig.6. It expresses a linear increase with $\frac{\Delta \omega_{S}}{\omega_{S}}$, repeated in accordance with amplitude minima shown in Fig.5.



Fig.6. Relative phase determination error depending on $\frac{\Delta \omega_{\mathrm{S}}}{\omega_{\mathrm{S}}}$ for

$$
N=50
$$

The phase determination error is different in case of the two-phase lock-in method where the in-phase and the quadrature component are used for phase determination. In case $\omega_{R}=\omega_{S}$, the real component of the two-phase system is proportional to $\cos (\varphi)$ and imaginary to $\sin (\varphi)$ and $\arccos \left(\left.2 \overline{u_{1}(t)}\right|_{\omega_{R}=\omega_{S}}\right)=\arcsin \left(\left.2 \overline{u_{2}(t)}\right|_{\omega_{R}=\omega_{S}}\right)$.

In case $\omega_{R} \neq \omega_{S}$ we analyze the term

$$
\begin{aligned}
& \delta\left[^{\circ}\right]=\left.\phi_{1}\right|_{\omega_{R} \triangleright \omega_{S}}-\left.\phi_{2}\right|_{\omega_{R} \triangleright \omega_{S}}= \\
& =\arccos \left(\left.2 \overline{u_{1}(t)}\right|_{\omega_{R}>\omega_{S}}\right)-\arcsin \left(\left.2 \overline{u_{2}(t)}\right|_{\omega_{R}>\omega_{S}}\right)
\end{aligned}
$$

representing the difference between the in-phase and the quadrature component. Fig.7 demonstrates this difference that depends on the term $\frac{\Delta \omega_{S}}{\omega_{S}}$ and on the number of periods and the phase difference between the input and the reference signals. The difference is zero only in one case when the frequencies are synchronized. This principle can be used to accurately synchronize the signals.

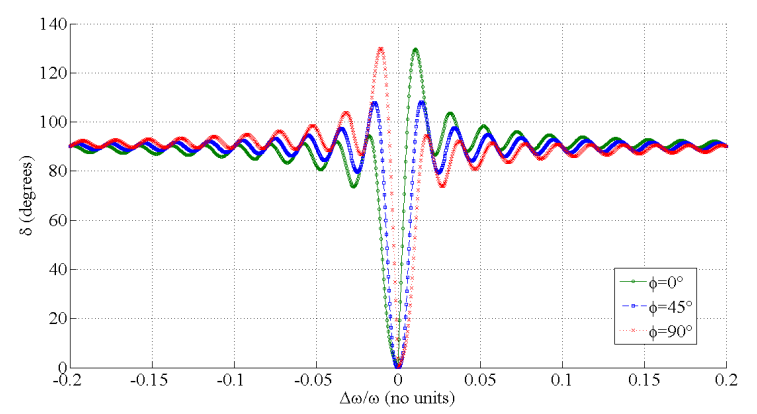

Fig.7. Phase difference between real and imaginary component of a two phase lock-in system.

\section{IMPEDANCE DETERMINATION}

\subsection{Theoretical analysis and numerical results}

In the previous chapter we described the use of a lock-in method for a single input signal. In the present chapter we analyze the impedance method for which we analyze two signals: the input signal in the (bio)impedance method is the current $i(t)$, while the output signal is voltage $u(t)$ in accordance with the measurement scheme shown in Fig. 10. For theoretical analysis we assume both signals are ideal sinusoidal, having the same frequency, but differ in phase and amplitude. Both signals are analyzed using the lock-in method as described, to obtain the amplitude $I_{S}$ and $U_{S}$ as well as the phase $\phi_{I}$ and $\phi_{U}$. As shown in the previous chapter both amplitude and phase depend on the frequency of the reference signal and the number of periods of the signals used. However, in case both signals are compared to the same reference signal (as they are), then the shape of the amplitude variation with the reference signal is the same for both signals as presented in Fig.8. The discrepancies occur only in points with very small amplitudes (in theory at amplitude zeros) that are due to rounding errors.

Fig.9 presents phase dependence on the frequency of the reference signals for both signals as well as its difference. The phase difference is equal, disregarding the frequency of the reference signal except in points of zero amplitudes. Numerical analysis was carried out at $N=20, U_{R}=1 \mathrm{~V}, U_{S}=1 \mathrm{~V}, I_{S}=1 \mathrm{~A}$ and phase difference $\phi=\phi_{U}-\phi_{I}=0,27^{\circ}$ for better presentation. 


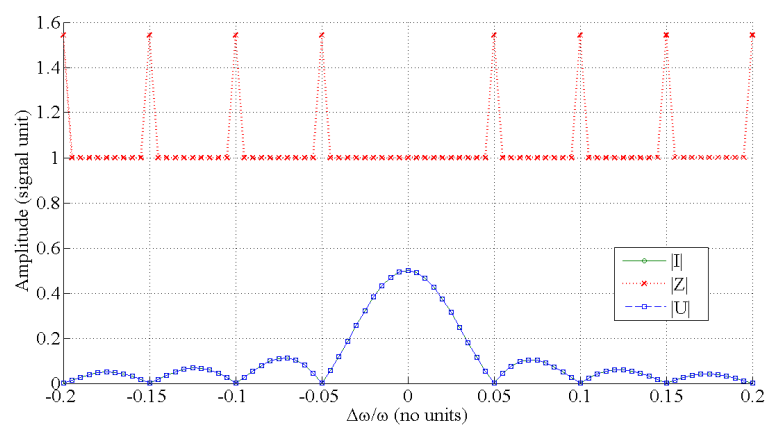

Fig.8. Amplitude $(|I|,|U|)$ and impedance magnitude ( $|Z|)$ determination depending on $\frac{\Delta \omega_{\mathrm{S}}}{\omega_{\mathrm{S}}}$ for $N=20$.



Fig.9. Phase $\left(\varphi_{I}, \varphi_{U}\right)$ and impedance phase $(\varphi)$ determination depending on $\frac{\Delta \omega_{\mathrm{S}}}{\omega_{\mathrm{S}}}$ for $N=20$.

\subsection{Experimental analysis and measurement results}

Experimental verification was performed on previously described prototype device using several test impedances (Fig.10). Device design is based on physical separation of the measurement and the data parts shown in Fig.10. The measurement component is used for acquiring voltage and current wave forms from device under test and is based on usage of a low cost microcontroller with built-in $\mathrm{AD} / \mathrm{DA}$ converters [9]. Data are sent to the computer for further reconstruction, analysis and presentation. The communication between the data part (computer) and measurement part (device) is established through an Ad-Hoc WiFi connection [17]. Data part uses a digital lock-in method (algorithm) for reconstruction of the amplitude and phase of the excitation and output signals and further determination of the magnitude and phase of the (bio)impedance. The frequency of the excitation and output signals is determined more exactly in a post-processing step from digitized signals. Frequency of the reference sine signal used in digital lock-in method should in theory be equal to the frequency of the excitation signal in order to avoid errors in determination of the amplitude and phase of the excitation and output signals [3].

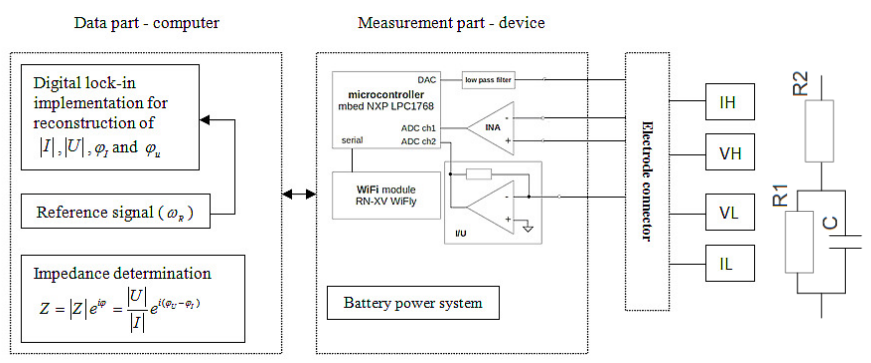

Fig.10. Measurement scheme for (bio)impedance determination and impedance under test.

The synchronization test was performed on the RC circuit with nominal values of $R_{1}=33 \Omega, C=100 \mathrm{nF}$ in $R_{2}=1 \mathrm{k} \Omega$ at signal frequency $f_{S}=1 \mathrm{kHz}$. Frequency $f_{R}$ of reference sine signal used in digital lock-in was generated in the range from $800 \mathrm{~Hz}$ to $1200 \mathrm{~Hz}\left(f_{R}=f_{S}+\Delta f_{S}\right)$.

Signal reconstruction and impedance determination was analyzed in the reference frequency range and presented in dependency on the ratio $\frac{\Delta \omega_{\mathrm{S}}}{\omega_{\mathrm{S}}}$. Results of impedance magnitude and phase determination are shown in Fig.11 and Fig.12.

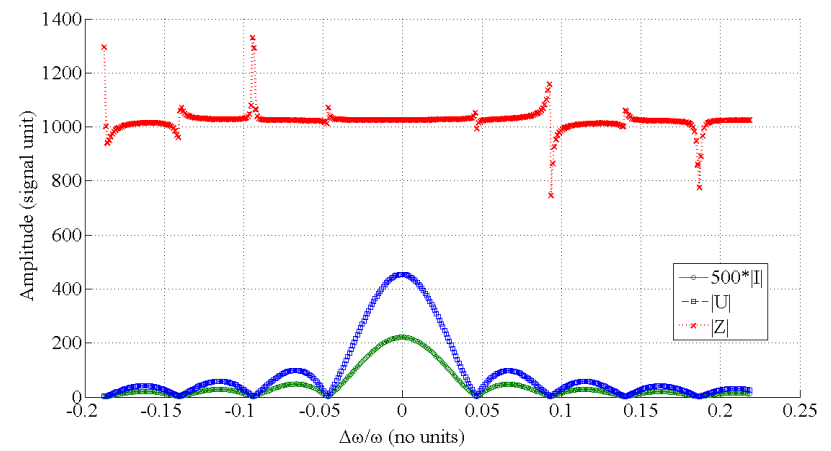

Fig.11. Amplitudes of a current and voltage signals depending on the ratio $\frac{\Delta \omega_{\mathrm{S}}}{\omega_{\mathrm{S}}}$.

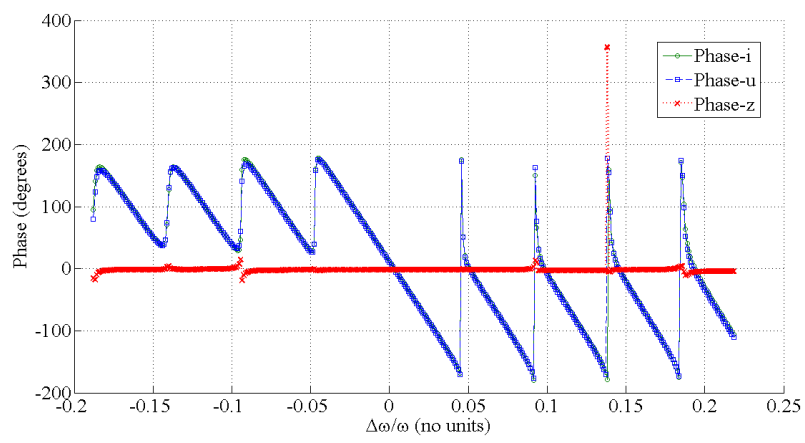

Fig.12. Phase of a current and voltage signal and its difference depending on the ratio $\frac{\Delta \omega_{\mathrm{S}}}{\omega_{\mathrm{S}}}$. 


\section{DISCUSSION}

Although the general idea behind phase sensitive detection or lock-in method is straightforward, additional issues should be considered when it comes to the actual implementation. These include frequency synchronization, limited accuracy of AC/DC converters, frequency drift, jitter, extrinsic/intrinsic noise, etc. Frequency drift and jitter are two different sources of error for a timing device. Jitter refers to variation in the output signal or its precision. A timing source with low jitter will output a relatively consistent frequency. Drift, in contrast, describes the change in accuracy of a given frequency over environmental changes such as temperature, humidity, pressure or simply changes over long periods of time. Low DAC-resolution, jitter and stochastic noise will affect signal-to-noise ratio of each measured signal, which can be improved by averaging. However, in case fast impedance measurements are required, this is possible only by reducing the number of sampled periods per frequency. In this case the influence of jitter cannot be canceled completely.

The lock-in method is typically used to improve the signal-to-noise ratio; in (bio)impedance the technique is used to perform signal reconstruction - determination of impedance phase and modulus. Several modern bioimpedance systems separate the measurement and data processing parts. In such cases the input and the reference signals are not generated from the same clock source so their frequencies may differ. Hence, a method of synchronization of a reference and input signal may be required to avoid the potential emergence of digital phase locked loops. In order to test the frequency synchronization, the best method seems to be to compare the in-phase and the quadrature component of the input signal as shown in Fig.7. Only when the frequencies are synchronized the phase difference is zero.

Significant errors in phase and amplitude of a signal can occur in case the reference and input signal are not synchronized. Such errors depend on the relative frequency difference and number of integration cycles.

From the viewpoint of rejecting asynchronous noise and achieving better signal-to-noise ratio in signal reconstruction it is crucial to reduce signal bandwidth (2). The price to be paid, however, is an excessively long data acquisition time, during which the stability condition might be violated. If we look from the viewpoint of error determination due to nonsynchronized frequency of the reference and the analyzed signal, we notice that error sensitivity (slope) is increased with the number of integrated periods. The synchronization accuracy is thus more important at longer integration times.

If we want to avoid the errors due to non-integer number of integration cycles, care must be taken to fulfill the equation $k=N \frac{f_{s}}{f}$, where $k$ is number of samples, $N$ is number of sampled periods, $f$ is signal frequency and $f_{S}$ is the sampling frequency. If the values of the variables are whole numbers, each sample has its opposite pair and the average value of alternate components will be zero. Otherwise an error is entered into the measurement system that is more pronounced for small number of integrated periods.

The impedance modulus is obtained by determination of the quotient of the amplitudes of both signals while the impedance phase is determined from the phase difference. The ratio of amplitudes yields the same results regardless of the frequency of the reference signal. In theory it is thus not important to have absolutely equal frequency of the reference and analyzed signals because the ratio between the amplitudes is the same. The same holds for the phase difference between the signals which (in theory) does not depend on the frequency of the reference signal. In both cases, however, the results are incorrect in points of zero or very small determined signal amplitudes (in theory at amplitude zeros) that are due to rounding errors. These deviations are dependent on the number of integrated periods and the phase difference between the signals. In numerical analysis using pure sine signals, deviations are seen as error spikes (Fig.8 and Fig.9). If, however, we included in analysis a quantization (number of bits) error and asynchronous noise (not shown in figures), the errors are dispersed around the spikes (Križaj D, data not shown). The same outcome was observed in experimental analysis, where the signal was created with only 10 bit resolution and the data acquisition was prone to jitter and other sources of noise.

Experimental analysis was carried out in the frequency range from $1 \mathrm{kHz}$ to $40 \mathrm{kHz}$. Variable $\frac{\Delta \omega_{\mathrm{S}}}{\omega_{\mathrm{S}}}$ was varied in the same ratio regardless of the analyzed signal frequency so impedance modulus and phase determination had a similar course at higher frequencies, but different values. Clock frequency of a microcontroller may vary in time and introduce frequency deviations in signal reconstruction, which in turn also affect the form of the determination error. In theory amplitude zeros of both signals appear in exactly the same points, which is not the case in experimental analysis where the errors are becoming more pronounced at higher values of $\frac{\Delta \omega_{\mathrm{S}}}{\omega_{\mathrm{S}}}$.

The averaging method might be interesting to increase the accuracy of magnitude and phase determination or to verify the correctness of the method.

\section{CONCLUSION}

Synchronization errors of the lock-in method, also known as phase sensitive detection, were analyzed by theoretical investigation, experimentally using an approach based on comparison of the investigated signal to a reference signal by correlation. When the frequency of the reference signal is equal to the frequency of the investigated signal, the amplitude and phase of the investigated signal can be determined simply from the result of integration of the product of the reference and the investigated signal (correlation). The output is proportional to the amplitude and cosine of the phase difference between the signals. If the frequencies of the signals differ, which can occur in case the frequency of the investigated signal is not exactly known, an 
error in amplitude and phase determination occurs. The amount of this error depends on the relative difference between the frequencies of the signals and the number of periods used in integration. The phase error is zero only at synchronized frequencies when the correlation function is maximal. In search for frequency synchronization we do not know in advance the expected maximal value of correlation so phase error is a better sign of non-synchronization between the signals.

When the lock-in method is used in the (bio)impedance measurement method, two (current and voltage) signals are concomitantly analyzed using the in-phase and the quadrature components. The impedance modulus is obtained by determination of the quotient of the amplitudes of both signals while the impedance phase is determined from the phase difference. It is shown that in this case the frequency synchronization is not mandatory so the impedance phase and modulus are correctly determined whilst disregarding signals that are not frequency synchronized. Experimental verification (a numerical investigation) shows that due to limited accuracy of the AC/DC converters and other sources of noise, a determination error necessarily exists in points of very small convolution amplitudes.

In summary, our study may aid in understanding the sources of impedance determination error when the lock-in method is utilized. In particular, our approach highlights the errors caused by the non-synchronized frequencies of the reference and the analyzed signals.

\section{REFERENCES}

[1] Blair, D.P., Sydenham, P.H. (1975). Phase sensitive detection as a means to recover signals buried in noise. Journal of Physics E: Scientific Instruments, 8, 621627.

[2] Buckingham, M.J. (1985). Noise in Electronic Devices and Systems. Ellis Horwood Ltd.

[3] Darren, W. (2007). Implementing digital lock-in amplifiers using the dsPIC®DSC. AN1115. Microchip Technology. http://ww1.microchip.com/downloads/en/ AppNotes/01115A.pdf

[4] Gabrielli, C. (1981). Identification of electrochemical processes by frequency response analysis. Technical report 004/83. Solartron Instrumentation Group.
[5] Grimnes, S., Martinsen, O. (2000). Bioimpedance and Bioelectricity Basics, Academic Press.

[6] Horowitz, P., Hill, W. (1996). The Art of Electronics, Second edition. Cambridge University Press.

[7] Iacopini, E., Smith, B., Stefanini, G., Carusotto, S. (1983). Digital techniques applied to phase-sensitive detection. Journal of Physics E: Scientific Instruments, $16,844-847$.

[8] Križaj, D., Baloh, M., Brajkovič, R., Žagar, T. (2013). Design and development of a portable WiFi enabled BIA device. Journal of Physics: Conference Series, 434, 012060.

[9] NXP Semiconductors N.V. (2014). LPC1769/68/67/66/65/63/63. Product data sheet. http://www.nxp.com/documents/data_sheet/LPC1769 68_67_66_65_64_63.pdf

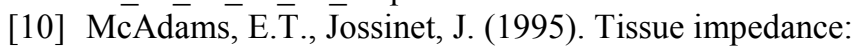
A historical overview. Physiological Measurement, 16, A1-A13.

[11] Meade, M.L. (1982). Advances in lock-in amplifiers. Journal of Physics E: Scientific Instruments, 15, 395403.

[12] Scofield, J.H. (1994). A frequency-domain description of a lock-in amplifier. American Journal of Physics, 62 (2), 129-133.

[13] Stanford Research Systems. About lock-in amplifiers. Application note \#3. http://www.thinksrs.com/ downloads/PDFs/ApplicationNotes/AboutLIAs.pdf

[14] Tarchanidis, K.N., Soilemes, A.T. (2011). Digitally synthesized alternative current sinusoidal waveform for resistive sensor measurement. Journal of Engineering Science and Technology Review, 4 (1), 50-54.

[15] Taub, H., Schilling, D.L. (1971). Principles of Communication Systems. McGraw-Hill.

[16] Rowing Networks. (2011). WiFi RN-XV-DS v0.3 8/18/2011. Data sheet. www.rovingnetworks.com/ files/resources/WiFly-RN-XV-DS.pdf

[17] Wilmshurst, T.H. (1990). Signal Recovery from Noise in Electronic Instrumentation. CRC Press.

[18] Raouf, A. (2013). Minimize frequency drift in crystals. Electronic Design, http://electronicdesign.com/analog/ minimize-frequency-drift-crystals.

Received July 02, 2014. Accepted October 30, 2014. 